\title{
Asphalten-Resin-Paraffin Deposits (ARPD) and Its Impact on Oil and Gas Production
}

\author{
${ }^{1}$ Liya H. Fokeeva, ${ }^{2}$ Ramilya Kimetova \\ ${ }^{1-2}$ Kazan Federal University \\ Email:fokeeva.00@mail.ru
}

Received: 20 ${ }^{\text {th }}$ August 2019, Accepted: $3^{\text {th }}$ September 2019, Published: $3^{\text {st }}$ October 2019

\begin{abstract}
This article discusses the implementation of a comprehensive program for the technical re-equipment of oil and gas production. The methods of combating the formation of ARPD in wells, intensive introduction of new equipment and technology, automation tools and automated production control systems into production were analyzed and reviewed. Based on the analysis, it was justified the use of acceptable methods to reduce the formation of ARPD.

Due to the hydrocarbon content, we noted the effect of paraffin wax and asphaltene on a decrease in the corrosion rate. Despite this, the formation of paraffin wax and paraffin asphalt creates operational difficulties. Paraffins are organic molecules that dissolve in crude oil at higher temperatures in reservoir conditions, but are deposited in pumps, rods, pipelines and storage tanks, where operating temperatures are relatively low. Paraffin dissolving solvents are mixtures of organic solvents and surfactants. Paraffin inhibitors are chemicals that inhibit the growth of paraffin crystals. They are more effective when applied before the formation of paraffin crystals. The bonding element in paraffin is asphaltene. Paraffin dispersants act on asphaltenes (binders) and reduce their ability to bind to the surface. They are used as dispersion in water or are injected directly into the aqueous phase in a well, pipelines and reservoirs.
\end{abstract}

Keywords

ARPD, Well, Automation, Reservoir, Production, Oil, Gas

\section{Introduction}

During the implementation of the program, the development of the oil and gas industry provides for the massive use of unified schemes for the collection, transport, preparation and storage of oil and gas. The technical re-equipment of the oil and gas production industry and its comprehensive automation made it possible to increase oil and gas production at an unprecedented pace [1]

Heavy oil and bitumen are widely used as additives to crude oil as raw materials for the production of liquid fuels, as well as for the production of many other products, as well as ubiquitous plastics.

From a chemical point of view, heavy oil and bitumen are extremely complex mixtures of hydrocarbon compounds with significant amounts of nitrogen-containing, oxygen-containing and sulfur-containing components, as well as trace amounts of metal-containing components. As a result, various production processes are used and developed for the extraction of heavy oil and bitumen. However, the technologies and services used to extract conventional crude oil are limited to the use of heavy oil and tar sands.

Mathematical and engineering models developed for conventional oil production are generally not used for the production of heavy oil and bitumen. In addition, the high molecular weight part of heavy oil and bitumen consists of compounds (and not just asphaltic components) with high melting points and high pour points, which significantly reduces displacement compared to conventional oil under various conditions.

The flow of traffic (heavy oil, possibly with sand, water, natural gas and/or solvents, in pipelines or horizontal wells) shall be predicted and guaranteed.

The examples of adverse effects that affect flow control: suspended sand may precipitate from the fluid and block flow in pipelines or long horizontal wells; deposits of asphaltene components in tubing or piping are also possible; the lower temperatures that prevail in the overlying rocks, in the Arctic regions, underwater and northern pipelines, may require heating elements, addition of solvents, insulated pipes and other flow methods. There is also a need to monitor the composition of heavy oil or bitumen as a means of assessing potential for improving on-site quality. One approach is to check produced fluids or use the on-site fluid monitoring method, either with casing logging, well sampling, or constant sensors.

In addition, it is important to recognize that a property such as viscosity (sometimes referred to as severity) of heavy oil bitumen and tar sand is mainly the result of an internal balance between the relatively high proportion of complex high molecular weight non-paraffin compounds and the low fraction of volatile compounds with lower molecular weight. The problems of producing heavy oil from a formation or bitumen from a field are usually the result of an imbalance in internal balance, which, in turn, affects material mobility, in addition to the deposition of asphaltene components. Success in the recovery of heavy oil and bitumen depends on both understanding the properties of these materials and knowledge of the formation geology. The reason is that the chemical differences between heavy oil (and bitumen from tar sand) and conventional oil ultimately affect their viscosity and, therefore, their mobility and recovery degree. 


\section{Methods}

As a result of the discovery and development of oil and gas fields in Tatarstan, Siberia and other regions, very difficult tasks were set for scientists and practitioners of our country. In the process of field development, the scientists made a fundamental contribution to the theory and practice of fundamentally new methods of development, using intensive waterflooding methods and injection wells. Intensive methods have found application not only in large deposits, but also in small-sized deposits characterized by poor filtration conductivity of the reservoirs. A significant contribution by Soviet scientists was made to the theory and practice of developing and operating oil and gas fields in permafrost. ajor works are being done in our country on the development of methods for intensifying the influx of oil and gas to wells, and on increasing the oil and gas recovery of reservoirs. At the same time, oil and gas industry workers still face many unsolved problems in increasing the efficiency of operation of oil and gas deposits. The challenge is to increase oil and gas recovery. [2]

Currently, recoverable oil reserves do not exceed $45-50 \%$ of absolute. Significant amounts of gas and condensate remain in the bowels of the earth. Much remains to be done on the utilization of all gas produced with oil, on the automation of oil and gas facilities and on the introduction of an automated control system (ACS) for the oil and gas industry. It is necessary to take measures to increase the residual reserves from the numerous old oil deposits.

Oil production is accompanied by the inevitable change in thermodynamic conditions and the transition of oil from reservoir to surface conditions. At the same time, temperature and pressure decrease.The phase equilibrium of individual hydrocarbons in the mixture is violated and they are released in the form of hydrocarbon gases and solid, or ointment-like heavy fractions in the form of paraffin, resins, asphaltenes. [3]

The production of heavy oil and bitumen can be optimally designed by carefully evaluating the quality of these materials using standard test methods that allow understanding their chemical and physical properties (Speight, 2015 Speight, 2014b, 2014c).). Assessment schemes shall not be complicated, but shall focus on the key parameters that affect recovery and can be determined by a number of features that affect the recovery of heavy oil or bitumen.

The relationship between the deposition rate and the shear rate, i.e., the shift of paraffin molecules, is due to the hydrodynamic resistance of the fluid, which depends on the flow rate and fluid viscosity. Higher viscosity and lower flow rates lead to high paraffin deposition rates, but paraffin deposition rates decrease with increasing flow under highly turbulent flow conditions, because paraffin is mechanically cut off from deposits on the pipe wall. Shear rate increases with increasing thickness of the deposit due to a decrease in flow area and an increase in flow rate, which is reflected in a decrease in the deposition rate of paraffin.

On the other hand, molecular diffusion is a process in which a radial temperature gradient in a line induces a concentration gradient of dissolved paraffin components in the liquid phase. This concentration gradient causes paraffin to diffuse toward the pipe wall, where it is expected to precipitate. Widely recognized transport methods that contribute to the wax thickness on the pipe wall are molecular diffusion of the dissolved wax, particle transport of the deposited wax, and exfoliation of the previously deposited wax.

As for chemical treatment, there is a need to remove paraffin wax, which is deposited from the waxy crude oil (fouling with paraffin). The compounds for paraffin processing are used to reduce oil viscosity when it is cooled while passing through the pipeline to maintain full throughput in the pipeline, to minimize paraffin deposition on the pipe walls, which causes a decrease in diameter.

The predominant types of paraffin-controlling compounds are crystal modifiers and dispersants. Crystal modifiers (depressants, flow improvers) distort the growth and three-dimensional shape of paraffin crystals, and paraffin deposits occur in the form of small round particles, rather than needle-like crystals - the latter types of crystals adhere and form gels that significantly increase the oil viscosity. Thus, crystal modifiers change the shape of crystals, which are less likely to attach to pipe walls or other wax crystals. In addition, the size of such crystals remains small, and the crystals are less prone to sedimentation and agglomeration.

Dispersants are surface-active compounds (surfactants) that change the surface energy of paraffin crystals and thereby change the interaction energy (interfacial energy) of a paraffin crystal and oil and adversely affect the probability of precipitation of crystals on the pipeline walls - crystals are also less likely to interact with each other and remain dispersed in the oil.

Fraction analysis also provides additional information on the intramolecular relationships of oil, again with an emphasis on the potential for precipitation of asphaltenes and possible thermal changes that can affect the stability of heavy oil or bitumen and cause an undesirable effect on the deposition of solids during recovery. In addition, useful information can be obtained from knowledge of elemental composition and molecular weight, as well as profiles of size exclusion chromatography and high performance liquid chromatography (HPLC).

Fine paraffin particles can remain in suspension and can be carried away by the fluid stream. Under certain conditions, they stick together with asphaltenes and resins, forming small lumps of hydrocarbons that adhere to the rough walls of the pipes, reducing their cross-section. The beginning of ARPD is noted at depths of 900-300 meters from the wellhead. Low flow rates and periodic exposure of the pipe surface due to pulsation contribute to paraffin deposition.

The ARPD formation of occurs mainly in wells operating in a batch mode or an accumulation mode, as well as in wells with a low flow rate. 
Flushing with hot oil to remove the ARPD wax is ineffective, or little effective, since the oil manages to cool when pumped into the pipes to the required interval, and unremoved paraffin crystals create crystallization centers around which the ARPD forms very quickly again .[4] [5]

\section{Results}

The most effective removal of ARPD is an injection of paraffin inhibitors. Well protection with ARPD inhibitors, given the presence of low dynamic levels, the periodic mode of well operation with pumping production and work with the accumulation of fountain wells, as well as low flow rates of most wells complicated by ARPD, shall be carried out by all protection methods, choosing wells in each case.

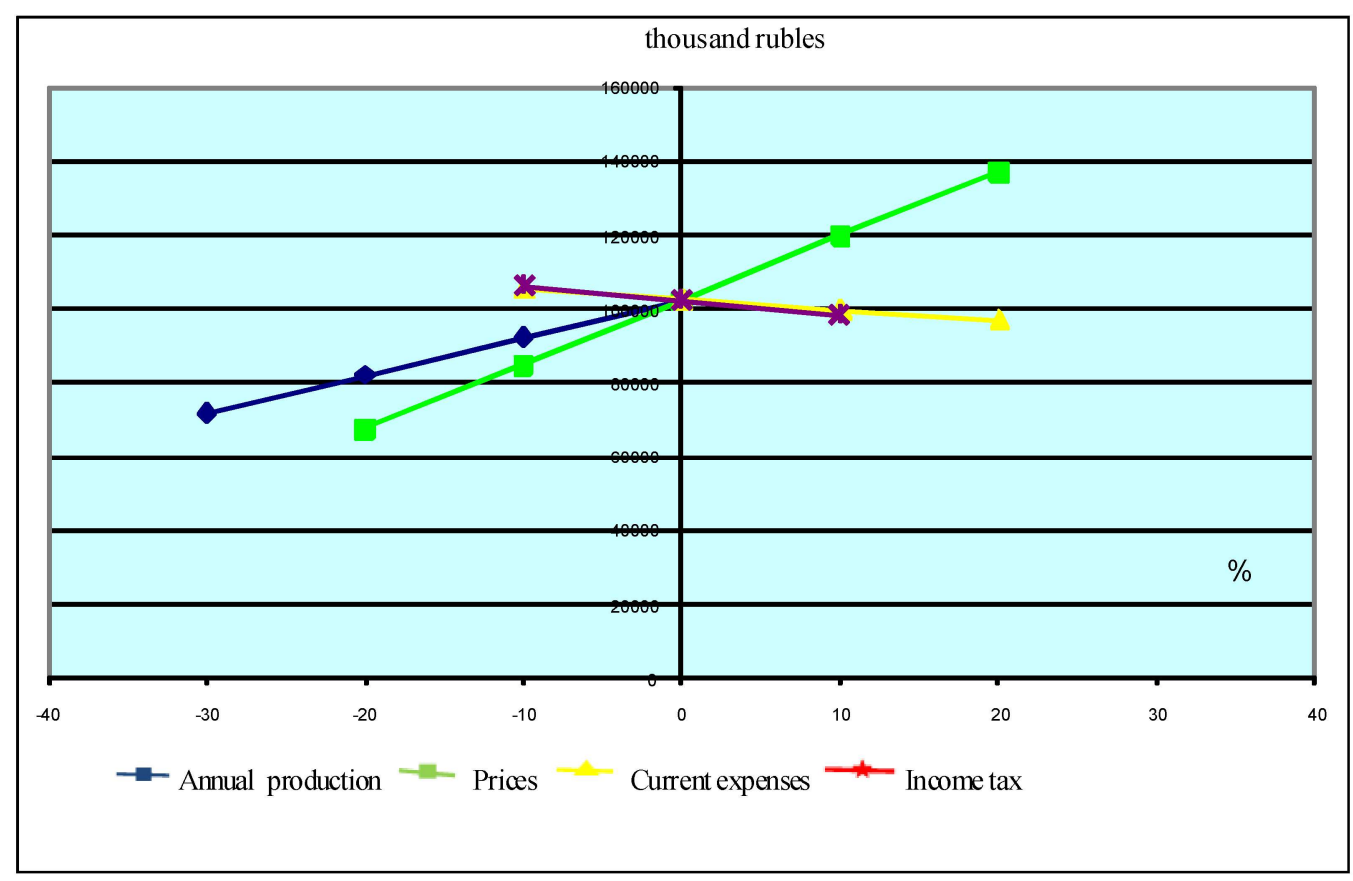

Fig.1: Chart "Spider"

In Fig. 1 the accumulated cash and net present value shows the dynamics of the receipt of funds to the account of the enterprise. The implementation of this event is economically viable, because it brings significant revenue to the enterprise at low cost. The location of the "spider" chart in the positive area indicates that it will bring the company only profit with any variation of factors.

\section{Conclusions}

Wells equipped with ESP with a low dynamic level are not recommended to be protected by the method of periodic injection of the inhibitor into the annulus, since the reagent is quickly removed by the product stream [6] [7]. Given the low dynamic levels and low permeability, most of the wells working with reservoirs are recommended to be protected by an inhibitor using the technology of continuous reagent supply using deep dosing units equipped at the wellhead. The use of inhibitors to protect oilfield equipment from ARPD increases the overhaul period of wells by several times. Promising developments of ARPD inhibitors tend to create complex inhibitors using biodegradable surfactants, which makes it possible to increase not only the inter-treatment period by 5 or more times, but also create conditions for initial, downhole oil demulsification, which in turn will reduce the demulsifier consumption and improve quality of oil preparation for refining.

It should be noted that the use of traditional methods of removing ARPD seemed inefficient due to their use in wells that do not meet the boundary parameters of their application. The action of acids, reagents and inhibitors of paraffin deposits are based on adsorption processes. When using chemical methods, they are based on the dosage of chemical compounds that reduce, and sometimes completely remove or prevent the ARPD formation.

Concerning the action basis of electric heaters, it should be noted that heating will occur over the entire interval from the bottom to the wellhead, thereby there will be no temperature difference during production; transportation of ARPD petroleum products will not be delayed in oilfield equipment.

While maintaining constant pressure in the well, the ARPD will not be deposited in oilfield equipment.

An insufficient study of each of the above methods does not allow an exhaustive analysis and the most effective way to remove ARPD in oilfield equipment at wells and oil reservoirs.

We can exclude just the experience of recent positive developments, namely, the biological removal of ARPD in wells. [8] [9] 
In the process of working with the existing well stock, it is necessary to use as many various methods to combat ARPD as possible.

Since each well has its own individual features of work, the removal methods in the production process shall be different.

In the process of ongoing work, the well stock in which the application of certain methods for removing ARPD is most effective shall be determined.

\section{Summary}

When considering all the methods and ways to combat ARPD, we can draw the following conclusion.

The use of chemical methods to protect equipment from ARPD and their removal has not been widely used due to the high cost of the reagent and the high cost of technology for their application. The cost of the reagent for the elimination of ARPD (SNPKH 7880) based on the calculation of the consumption of 6-7 $\mathrm{m} 3$ for one injection cycle at a cost of 15 thousand roubles per ton will require significant costs during the year. It is necessary to pay attention to the fact that the need for reagents is shown without taking into account the inter-treatment period of the wells, that is, one effect on the elimination of ARPD.

The use of a two-row elevator did not bring the expected effect on the expected scale, although wells were identified where flushing with hot oil through a two-row elevator is quite effective. The inexpediency of further work was caused for two main reasons:

1. The repair interval (RI) of the considered category of wells has not changed, and in some cases even decreased.

2. The well cleaning interval (WCI) almost did not change, and the cost of pipes and equipment for wellhead piping of the well is quite an expensive "pleasure" that does not pay off during operation.

Theoretically justified as the most effective and recommended method for removing ARPD with heating shells and in practice has proved to be an effective and inexpensive method for removing "dead" plugs and wells where "non-profit" was obtained. Such work is ongoing. The work involved three links for cleaning the tubing lift with a scraper (on an automatic winch), and one thawer. The majority of wells have the ARPD formation interval of up to 300 meters. This fund accounts for up to $70 \%$ of all wells with intensive formation of ARPD, and $30 \%$ of them have a depth of ARPD up to 100 meters.

- Wells with a formation depth of up to 500-700 meters make up 28\% of all, and a formation depth of more than 500 meters is about $1.5 \%$.

- The formation of ARPD in tubing up to a depth of 100 meters is often observed in wells idle or stopped for some reason, regardless of production rate and water cut.

- In the wells with an interval of up to 300 meters, the average production rate is $52 \mathrm{~m} 3 /$ day with a water cut of up to $15 \%$.

- In the wells with an interval of up to 500 meters, the average production rate is $45 \mathrm{~m} 3 /$ day with a water cut of up to $25 \%$.

Thus, we can conclude that the depth of ARPD formation is inversely proportional to the flow rate of wells.

\section{Acknowledgements}

The work is performed according to the Russian Government Program of Competitive Growth of Kazan Federal University.

\section{References}

1. Ibragimov N.G. et al. Complications in Oil Production. Ufa, 2003. - 302 p.

2. G.A. Mansoori. Paraffin/Wax and Waxy Crude Oil. The Role of Temperature on Heavy Organics Deposition from Petroleum Fluids.UIC/TRL Heavy Organics Deposition home page.

3. Sorokin S.A., Khavkin S.A.Features of the Physico-Chemical Mechanism of ARPD Formation in Wells // Drilling and Oil. No. 10.2007. - P. 30-31.

4. K. Arnold, M. Stewart. Surface production operations. 1999. - P. 55

5. Havard Devold. Oil and gas production handbook 2006. - P. 33

6. N.B. Tomson. Mechanisms of mineral scale inhibition/ N.B. Tomson, G. Fu, M.A. Watson, A.T. Kan // SPEF. 2003 Vol. 18 - No. 3 - P. 192199

7. J.K. Kerver, I.K. Heiheiker. Scale inhibition by the squeeze technique// J. Canada Petrol. Technol. 1969. - 1 Vol. 8 P. 15 - 23

8. J.K. Fink. Oil field chemical // A. - T.: GPP. 2003. - 495p.

9. V.N. Glushchenko et al. Oilfield Chemistry:Complications in the Reservoir Well System - UPPN / MAKS Press, 2008. -328 p. 